\title{
Diabetes in pregnancy: management of diabetes and its complications from preconception to the postnatal period (NG3)
}

\author{
JONATHAN WEBBER, ${ }^{1}$ MARY CHARLTON, ${ }^{1}$ NINA JOHNS²
}

\begin{abstract}
In February 2015 the National Institute for Health and Care Excellence (NICE) published new guidance (NG3) on the management of diabetes in pregnancy. Care teams need to be aware of this guidance and implement its recommendations. These include preconception care with target $\mathrm{HbA}_{1 \mathrm{c}}$ $48 \mathrm{mmol} / \mathrm{mol}$. Women at risk of gestational diabetes mellitus (GDM) should have a $75 \mathrm{~g}$ oral glucose tolerance test (OGTT). Diagnostic criteria for GDM have changed to fasting glucose of $5.6 \mathrm{mmol} / \mathrm{L}$ or above or $\mathbf{2}$ hour glucose of $\mathbf{7 . 8}$ $\mathrm{mmol} / \mathrm{L}$ or above.

Glycaemic targets in all diabetic pregnancies have changed to fasting glucose below $5.3 \mathrm{mmol} / \mathrm{L}$ (4-5.2 $\mathrm{mmol} / \mathrm{L}$ if on insulin) and 1 hour postprandial glucose below $7.8 \mathrm{mmol} / \mathrm{L}$ if these can be achieved safely. Continuous glucose monitoring and insulin pump therapy should not be used routinely but can be used if glycaemic control is problematic. Capillary ketone testing should be routine for women with type 1 diabetes when hyperglycaemic and for all women with diabetes including, GDM when acutely unwell.

More flexibility is offered around recommended delivery timing: 37+0 weeks to $38+6$ weeks for women with types 1 and 2 diabetes; prior to $40+6$ in GDM (and earlier if complications arise). Postnatal testing following GSM should be by fasting glucose (not OGTT) at 6-13 weeks post partum. Testing later than this can use $\mathrm{HbA}_{1}$.

Introducing these changes will have resource implications, including a likely increase in the number of women diagnosed with GDM.
\end{abstract}

Br J Diabetes Vasc Dis 2015:15:107-111

Key words: NICE guidance, NG3, pregnancy in diabetes, diabetes in pregnancy, pre-conception care, gestational diabetes, antenatal diabetes, postnatal screening

University Hospitals Birmingham NHS Foundation Trust, Birmingham, UK. Birmingham Women's Hospital NHS Foundation Trust, Birmingham, UK.

Address for correspondence: Dr J Webber

Consultant Physician, Diabetes Centre, Nuffield House, Queen Elizabeth

Hospital, Birmingham B15 2TH, UK

E-mail: jonathan.webber@uhb.nhs.uk

http://dx.doi.org/10.15277/bjdvd.2015.029

\section{Introduction}

The 2015 guidance from the National Institute for Health and Care Excellence (NICE) on Diabetes in Pregnancy (NG3, updated from 2008) provides a helpful template on which to base diabetes and pregnancy services. ${ }^{1,2}$ It is separated into six areas: preconception planning and care, gestational diabetes mellitus (GDM), antenatal care for women with diabetes, intrapartum care, neonatal care and postnatal care. New recommendations have been made in a number of areas, in particular around aspects of diagnosis of GDM and management and the use of newer technologies.

\section{Blood glucose and plasma glucose}

In this article we shall often refer to both plasma glucose (in, for example, glucose tolerance testing) and capillary glucose (in selfmonitored blood glucose testing) simply as "glucose". This recognises that the term "blood glucose", although in common parlance, usually refers to capillary glucose levels measured by meters calibrated to plasma glucose equivalents.

\section{Preconception planning and care}

There is now a much stronger emphasis on preconception planning and care and on ensuring that appropriate information is imparted to all women of childbearing age. The importance of avoiding an unplanned pregnancy should be included in education from adolescence for women with diabetes. Guidance on contraception is provided, with reassurance that oral contraceptives can be used safely by women with diabetes. Women/couples planning a pregnancy should be educated on what to expect in terms of the impact of diabetes on pregnancy (including the necessity of frequent contact with health professionals during the pregnancy). Moreover, women with diabetes who are planning to become pregnant should be told that establishing good blood glucose control before conception and continuing this throughout pregnancy will reduce the risk of miscarriage, congenital malformation, stillbirth and neonatal death. It is important to explain that risks can be reduced but not eliminated.

It is not clear whether the information provided should include absolute as well as relative risks. It is correct that diabetes doubles the risk of having a baby with a major malformation (an increase in risk from about 21/1000 births to 42/1000 births ${ }^{3}$ ), but this can be overemphasised. Most women will have a good outcome and it is also important to impart this information, while placing emphasis on the benefits of improving their glycaemic control.

We await confirmation of pre-pregnancy glucose targets, which 
will be included within the NICE guidance for the management of type 1 diabetes that is due out in late 2015. The draft available on the NICE website suggests aiming for pre-breakfast glucose of 5-7 $\mathrm{mmol} / \mathrm{L}$ and $4-7 \mathrm{mmol} / \mathrm{L}$ before other meals. The guidance also includes a post-meal target of 5-9 mmol/L, though it does not provide the timings after meals when blood glucose should be measured.

The preconception $\mathrm{HbA}_{1}$ target has been amended to a more realistic value of $<48 \mathrm{mmol} / \mathrm{mol}$ and recognises any reduction towards this as helpful (the 2008 target of $<43 \mathrm{mmol} / \mathrm{mol}$ was almost unobtainable for many patients). Monthly $\mathrm{HbA}_{1 \mathrm{c}}$ measurements continue to be recommended in the preconception stage, although this may not be appropriate for some women who may spend years trying to conceive, particularly those who have achieved a glycaemic target and whose clinical condition is stable.

The offer of blood ketone testing strips to those with type 1 diabetes (with provision of appropriate education) is sensible, given the increased emphasis on ketone testing in those who are unwell during pregnancy. Targets for action points need to be developed both in the preconception period and during pregnancy. For example, before conception, patients should be educated to test for ketones if glucose is above $13 \mathrm{mmol} / \mathrm{L}$ and to use corrective doses of insulin if ketones are above $1.5 \mathrm{mmo} / \mathrm{L}$, as per the Dose Adjustment For Normal Eating (DAFNE) programme. Lower ketone levels during pregnancy may trigger actions. Testing for ketonaemia is urgent if a pregnant woman with any form of diabetes presents with hyperglycaemia or is unwell, in order to exclude diabetic ketoacidosis.

There is no change to advice around the safety of medicines used in the prevention and management of complications of diabetes. Both angiotensin converting enzyme inhibitors and angiotensin II receptor antagonists should be discontinued before conception or as soon as pregnancy is confirmed. We are aware that many clinicians would continue these until pregnancy was confirmed in patients with significant nephropathy. Such patients will usually be under the care of a nephrologist. Indeed, the guidance advises that those with either estimated glomerular filtration rate $<45 \mathrm{~mL} / \mathrm{min} /$ $1.73 \mathrm{~m}^{2}$ or urinary albumin:creatinine ratio $>30 \mathrm{mg} / \mathrm{mmol}$ should be referred to a nephrologist prior to conception.

\section{Gestational diabetes}

Gestational diabetes and adverse pregnancy outcomes

GDM is glucose intolerance first diagnosed in pregnancy and expected to resolve after pregnancy. As NG3 says, it brings an increased risk of fetal macrosomia, birth trauma, neonatal hypoglycaemia, perinatal death, induction of labour, Caesarean section, childhood obesity and, above all, future maternal type 2 diabetes. NICE provides no advice on the extent to which these risks are reduced by management of gestational diabetes and advises counselling women that GDM carries a "small increased risk of serious birth complications such as shoulder dystocia". In contrast, the seminal Australian Carbohydrate Intolerance Study in Pregnant Women suggested a reduction in serious perinatal complications from $4 \%$ to $1 \%$ with treatment of GDM. ${ }^{4}$

The diagnosis and management of GDM have been plagued by inconsistent definitions and approaches to management. Those
Table 1 New plasma glucose thresholds for diagnosis of gestational diabetes mellitus (GDM) on $75 \mathrm{~g}$ oral glucose tolerance testing: only one threshold need be exceeded for diagnosis

\begin{tabular}{lll}
\hline & Normal & GDM \\
Fasting & $<5.6 \mathrm{mmol} / \mathrm{L}$ & $\geq 5.6 \mathrm{mmol} / \mathrm{L}$ \\
2-hour & $<7.8 \mathrm{mmol} / \mathrm{L}$ & $\geq 7.8 \mathrm{mmol} / \mathrm{L}$
\end{tabular}

of us who anticipated that this updated guidance would iron out these dilemmas will find much to celebrate but some outstanding quandaries.

\section{Diagnosing GDM: who, when and how?}

The new guidance, contrary to guidance from the International Association of Diabetes and Pregnancy Study Group (IADPSG), ${ }^{5}$ does not recommend universal testing for GDM, but retains testing with a $75 \mathrm{~g}$ oral glucose tolerance test (OGTT) at 24-28 weeks for women with a single risk factor. The advice has sensibly changed for women with a prior history of GDM, such that these women should either have an OGTT or begin self-monitoring blood glucose (SMBG) as early in pregnancy as possible. This will enable prompt identification and appropriate management of women who have developed unrecognised type 2 diabetes between pregnancies and also identify those who have impaired glucose tolerance and already fulfil the criteria for GDM. It appears that NICE is happy for centres to decide whether such diagnostic SMBG is for a fixed duration (a week, perhaps) or for the duration of the pregnancy. The former would save on costs and, arguably, maternal anxiety, while the latter would enable prompt intervention for incipient GDM.

NICE neither adopted IADPSG thresholds for diagnosing GDM nor retained the old World Health Organisation criteria. NICE guidance NG3 looked again at data from the Hyperglycemia and Adverse Pregnancy Outcomes study and at subsequent studies of the impact of IADPSG diagnostic criteria on pregnancy outcomes and performed an economic analysis appropriate to the National Health Service. ${ }^{1}$ Taking all of these into account, NG3 recommends (Table 1) that:

- Fasting glucose of $5.6 \mathrm{mmol} / \mathrm{L}$ or above is now diagnostic of GDM and/or

- 2 hour glucose (75 g OGTT) of $7.8 \mathrm{mmol} / \mathrm{L}$ or above remains diagnostic of GDM

These criteria are appropriate to England and Wales and are simpler than those of other countries, but diagnostic criteria remain inconsistent internationally (Table 2). 1,25-8

It will be a disappointment to many that NG3 does not offer advice on diagnosing GDM after 28 weeks of pregnancy. This should prompt us all to publish our data so the next revision of NICE guidance will have evidence on which to base advice in this controversial group.

\section{Targets and treatments for gestational diabetes}

The new guidance tightens up the initial management of GDM, stipulating the need for a consultation in the joint antenatal diabetic service within a week of diagnosis, prompt communication with primary care, and dietary advice specifically from a 
Table 2 International criteria for diagnosis of gestational diabetes

\begin{tabular}{|c|c|c|c|c|}
\hline Guidance & Process & Fasting threshold & Post-glucose load threshold(s) & Comments \\
\hline WHO $(2013)^{6}$ & \multicolumn{3}{|c|}{ As IADPSG } & \\
\hline $\mathrm{ACOG}^{7}$ & $\begin{array}{l}\text { Two step for all women; } \\
50 \mathrm{~g} \text { glucose challenge } \\
\text { followed by } 100 \mathrm{~g} \text { OGTT }\end{array}$ & $5.3 \mathrm{mmol} / \mathrm{L}$ & $\begin{array}{l}10 \mathrm{mmol} / \mathrm{L} \text { after } 1 \text { hour } \\
8.6 \mathrm{mmol} / \mathrm{L} \text { after } 2 \text { hours } \\
7.8 \mathrm{mmol} / \mathrm{L} \text { after } 3 \text { hours }\end{array}$ & $\begin{array}{l}\text { Only women with initial } \\
\text { glucose of } 7.5-7.8 \mathrm{mmol} / \mathrm{L} \text { or } \\
\text { above after } 50 \mathrm{~g} \text { glucose } \\
\text { challenge have OGTT. } \\
2 \text { abnormal levels diagnostic }\end{array}$ \\
\hline
\end{tabular}

dietitian focusing on a low glycaemic index diet. Thirty minutes of exercise after meals is also recommended.

Just as diagnostic thresholds have been reviewed, so have treatment targets (Table 3). The recognition that the majority of women with GDM will need blood glucose lowering medication confirms our own experience. What is less welcome is the continued requirement to obtain and document specific informed consent for prescribing metformin. NG3 firmly recommends metformin if diet and exercise have not controlled blood glucose within 1-2 weeks and the Metformin in Gestational Diabetes study established that the safety of metformin is comparable with that of insulin. ${ }^{9}$ It would be interesting to know how professionals are interpreting the need for consent and whether this deters women from taking metformin. Glibenclamide remains an option after 11 weeks' gestation for women who cannot tolerate metformin and/or decline insulin.

A new departure is the advice to start insulin, with or without metformin, immediately at diagnosis (with dietary advice but without a trial of diet alone) in women whose fasting glucose is $7.0 \mathrm{mmol} / \mathrm{L}$ or more and in women whose fasting glucose is $6.0-6.9 \mathrm{mmol} / \mathrm{L}$ if macrosomia or polyhydramnios is present (Table 3).

\section{Resourcing the new guidance for gestational diabetes}

NG3 anticipates that it will take just 5 minutes to explain to each woman the rationale and practicalities of testing for GDM and the OGTT outcome. This seems likely to be an underestimate, especially when the result is positive. Its prediction that testing can be absorbed within existing staffing ignores the obesitydriven rise in women requiring an OGTT. At Birmingham Women's Hospital we experienced a $45 \%$ rise in the number of OGTTs between 2012 and $2013 .{ }^{10}$ It also seems unrealistic to expect that reducing the (fasting) thresholds for diagnosis and intervention will not have a significant cost impact. Again, it is incumbent on those of us who work in the field to publish our data if we find our resources are stretched by NG3's guidance.
Table 3 New glucose thresholds for medication following diagnosis of gestational diabetes

\begin{tabular}{llll}
\hline & $\begin{array}{l}\text { Target for } \\
\text { self-monitored } \\
\text { glucose }\end{array}$ & $\begin{array}{l}\text { Stepwise } \\
\text { introduction } \\
\text { of metformin } \\
\text { and insulin } \\
\text { (self-monitored } \\
\text { glucose) }\end{array}$ & $\begin{array}{l}\text { Immediate } \\
\text { insulin } \\
\text { (diagnostic } \\
\mathbf{7 5} \text { results) }\end{array}$ \\
& $<5.3 \mathrm{mmol} / \mathrm{L}$ & $\geq 5.3 \mathrm{mmol} / \mathrm{L}$ & $\begin{array}{l}\geq 7.0 \mathrm{mmol} / \mathrm{L} \\
6.0-6.9 \mathrm{mmol} / \mathrm{L} \\
\text { if macrosomia } \\
\text { or } \\
\text { polyhydramnios } \\
\text { present }\end{array}$ \\
\hline Fasting & & & \\
\hline 1 hour post-meal & $<7.8 \mathrm{mmol} / \mathrm{L}$ & $\geq 7.8 \mathrm{mmol} / \mathrm{L}$ & \\
\hline hours post-meal & $<6.4 \mathrm{mmol} / \mathrm{L}$ & $\geq 6.4 \mathrm{mmol} / \mathrm{L}$ & \\
\hline OGTT: oral glucose tolerance test. & & \\
\hline
\end{tabular}

\section{Antenatal care}

The clear enumeration of the desired frequency of glucose testing for those on insulin therapy (whether type 1 diabetes, type 2 diabetes or GDM) will be helpful to guide patients and commissioners. We see significant numbers of patients who are unable to obtain sufficient test strips without attending for prescriptions on a very frequent basis. The guidance recommends testing seven times daily for those on multiple daily injection insulin regimes (fasting, pre-meals, one hour post-meals and prebed) and this does not allow for additional testing that may be required (e.g. related to driving, or overnight checking for hypoglycaemia). Even those patients not on insulin should be testing fasting glucose and one hour post-meal glucose. A separate costings document accompanies NG3, which suggests that 
recommendations on testing could potentially double the number of testing strips being used by pregnant women with type 2 diabetes or GDM. This will entail significant costs, as the document estimates that approximately $80-90 \%$ of women with type 2 diabetes and $20 \%$ of women with GDM are on a multiple daily insulin injection regimen. The costs appear to be underestimated, as the cost for a box of 50 testing strips is given as $f 2.29$. However, we are not aware of any test strips as inexpensive as this (apart from those testing for glycosuria). Most capillary testing costs about four times this amount, according to the NHS Electronic Drug Tariff.

Some changes have been made to glucose targets with a goal for fasting glucose to be $<5.3 \mathrm{mmol} / \mathrm{L}$ (previously $3.5 \mathrm{mmol} / \mathrm{L}$ to $<5.9 \mathrm{mmo} / \mathrm{L}$ ), but for those on insulin or glibenclamide to maintain this at $>4 \mathrm{mmol} / \mathrm{L}$. This is a challenging target and it is recognised that problematic hypoglycaemia may be a significant barrier to achieving it. In those on diet or metformin only, lower fasting glucose levels are acceptable and indeed are not infrequently seen in those with GDM.

It is suggested that $\mathrm{HbA}_{1 c}$ is measured in all women with GDM at the time of diagnosis, to identify those who may have pre-existing type 2 diabetes. Whilst potentially helpful in a few cases, this is likely to miss many patients with pre-existing diabetes as $\mathrm{HbA}_{1 c}$ falls during a normal pregnancy. ${ }^{11}$ In those with known pre-existing diabetes the recommendations are to consider measuring $\mathrm{HbA}_{1 \mathrm{c}}$ levels in the second and third trimesters, to assess the level of risk for the pregnancy, while not using $\mathrm{HbA}_{1 c}$ levels routinely during this period. These statements appear somewhat contradictory.

New technology comes to the fore with continuous glucose monitoring (CGM) being considered for pregnant women on insulin therapy who have problematic severe hypoglycaemia (with or without impaired awareness of hypoglycaemia), or who have unstable blood glucose levels (to minimise variability), or to gain information about variability in blood glucose levels. The main debating point will be whether funding is available for this (this has not been the case, in the absence of positive NICE guidance on this issue). Any applications for funding for CGM will need to be promptly answered by commissioners, unless separate funding is identified for CGM in tariff negotiations.

Ketone testing in those with type 1 diabetes is now widely accepted and should already be in place for those patients seen before conception. Additional guidance is that capillary ketone testing should now be performed in pregnant women with any form of diabetes if they present with hyperglycaemia (levels not defined), or are unwell, to exclude diabetic ketoacidosis. All maternity units will need facilities to conduct these tests, with staff trained in their use and interpretation.

The appropriate frequency of follow up during pregnancy is much debated. The guidance states that women with diabetes should have contact with the joint diabetes and antenatal clinic for assessment of blood glucose control every 1-2 weeks throughout pregnancy. This seems reasonable yet, within the separate costings statement, NICE suggests that only three additional clinic visits will be needed for women with a diagnosis of GDM. For a diagnosis around 26 weeks, with rapid review after diagnosis and subsequent follow ups, it is likely that five additional visits will be required.

Policies around the timing of delivery are variable; the guidance recognises this and allows for considerable variability. Those with previously diagnosed type 1 and type 2 diabetes and no other complications should have an elective birth by induction of labour, or by elective Caesarean section if indicated, between $37+0$ weeks and $38+6$ weeks of pregnancy. Bringing the delivery forward to 37 weeks is a little earlier than our current routine practice, where the window has been $38+0$ to $38+6$ weeks. Obstetricians will have to consider the potential use of antenatal corticosteroids for avoidance of transient tachypnoea of the newborn if considering elective delivery at 37 weeks' gestation by planned Caesarean section and balance the risks of potential maternal hyperglycaemia and inpatient monitoring with the risks of additional neonatal unit admissions.

Women with GDM are advised to give birth no later than $40+6$ weeks, and offered elective birth (by induction of labour, or by Caesarean section if indicated) if they have not given birth by this time. Elective birth is to be considered before $40+6$ weeks for women with GDM if there are maternal or fetal complications. Previously our practice has been to allow those women with well controlled GDM on diet alone to go to 40 weeks, but to induce women who have required either metformin and/or insulin therapy between $38+0$ and $38+6$. There will need to be local clarification around what counts as a "complication": does this include suboptimal glycaemic control in spite of therapy? Early elective birth had previously been recommended in GDM to reduce the risks of fetal macrosomia and birth trauma; therefore, macrosomia may now be considered as a complication, with a recommendation for birth before $40+6$ weeks.

\section{Neonatal care}

NG3 has left advice regarding care of the newborn infant of a woman with diabetes essentially unchanged, although there is some clarification of the schedule of care. The first feed should be within 30 minutes of birth and then feeds should be every 2-3 hours until the baby's glucose is maintained at $2.0 \mathrm{mmol} / \mathrm{L}$ or above. Intervention (intravenous or by tube) should be reserved for babies with glucose below $2 \mathrm{mmol} / \mathrm{L}$ on two consecutive occasions, abnormal clinical signs and/or poor oral feeding.

\section{Postnatal care}

There is no change in the advice surrounding management of women with pre-existing type 1 and type 2 diabetes immediately following delivery. The changes in follow up of women with GDM largely reflect the changes since 2008 in NICE guidance on the diagnosis of diabetes, in particular the use of $\mathrm{HbA}_{1 c}$ and prevention of diabetes.

Postnatal OGTT should not be used as a routine. Instead the recommendation is to use a fasting glucose 6-13 weeks after delivery, with $\mathrm{HbA}_{1 \mathrm{c}}$ used after 13 weeks if testing is delayed for some reason. Some will regret the loss of the OGTT and with it the opportunity to identify post-load dysglycaemia, while others will welcome the less complex approach.

Interpretation of the results is based on NICE guidance on preventing diabetes. ${ }^{12}$ A fasting glucose of $\geq 7.0 \mathrm{mmol} / \mathrm{L}$ or $\mathrm{HbA}_{1 \mathrm{c}}$ $\geq 48 \mathrm{mmol} / \mathrm{mol}$ requires confirmation to make a diagnosis of type 2 
Figure 1. Interpretation of postnatal testing following gestational diabetes

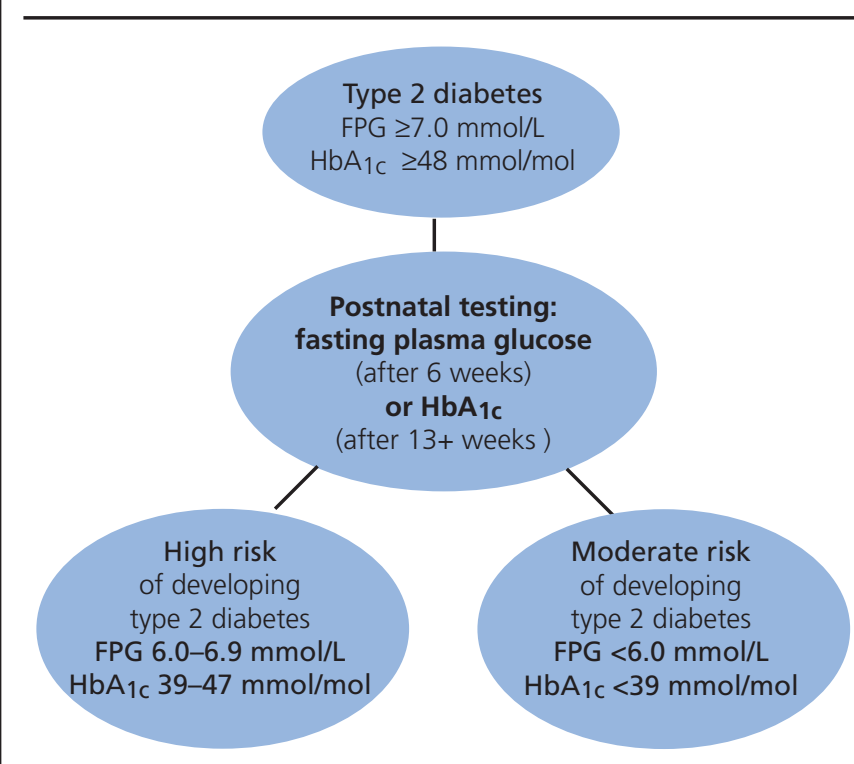

FPG: fasting plasma glucose

diabetes, with management in line with NICE guidance. Lower results are stratified as indicative of moderate or high risk of developing diabetes, as in Figure 1. All women whose postnatal test is not diagnostic for diabetes should have an annual $\mathrm{HbA}_{1 \mathrm{c}}$ and extra testing when planning future pregnancies.

\section{Conclusions}

The updated NICE guidance on Diabetes in Pregnancy contains a welcome combination of evidence-based and clinical common sense advice including preconception priorities, realistic antenatal targets, standardised diagnostic criteria, flexible deliveryplanning and simplified postnatal testing. Technology advice includes routine capillary testing but reserves more complex CGM for special cases. Gaps remain, notably with regard to prepregnancy targets and advice on late diagnoses of GDM. When these are revised in future, along with the resources required for a successful pregnancy in GDM, professionals must ensure their research and audit data are available to inform the next update.

\section{Conflict of interest None Funding None}

\section{References}

1. National Institute for Health and Care Excellence. Diabetes in pregnancy: Management of diabetes and its complications from preconception to the postnatal period. NG3, Feb 2015.

Available at https://www.nice.org.uk/guidance/ng3.

2. National Institute for Health and Care Excellence. Diabetes in pregnancy: Management of diabetes and its complications from pre-conception to the postnatal period. CG63, March 2008. Obsolete: no longer available online.

3. Confidential Enquiry into Maternal and Child Health. Pregnancy in women with type 1 and type 2 diabetes in 2002-03, England, Wales and

\section{Key messages}

- NICE has updated its advice on diabetes in pregnancy (NG3)

- Preconception care is to include target $\mathrm{HbA}_{1 \mathrm{c}}$ below $48 \mathrm{mmol} / \mathrm{mol}$

- Test for type 2 diabetes in early pregnancy where there is a history of previous gestational diabetes mellitus (GDM); otherwise around 26 weeks if "at risk"

- GDM is now diagnosed when fasting glucose is $5.6 \mathrm{mmol} / \mathrm{L}$ or above or 2 hour glucose is $7.8 \mathrm{mmol} / \mathrm{L}$ or above on a $75 \mathrm{~g}$ oral glucose tolerance test (OGTT)

- Glycaemic targets in pregnancy are now fasting glucose below $5.3 \mathrm{mmol} / \mathrm{L}$ (4-5.2 $\mathrm{mmol} / \mathrm{L}$ if on insulin) and 1 hour postprandial glucose below $7.8 \mathrm{mmol} / \mathrm{L}$ (4-7.7 $\mathrm{mmol} / \mathrm{L})$

- Increased use of capillary ketone testing is advised to avoid ketoacidosis

- Delivery should occur at 37+0 to 38+6 weeks for type 1 and type 2 diabetes, or before 41 weeks in GDM (earlier if there are complications)

- Postnatal testing following GDM should be with fasting glucose (not OGTT)

Northern Ireland. CEMACH: London; 2005. Available at http://www. hqip.org.uk/assets/NCAPOP-Library/CMACE-Reports/29.-2005-Pregnancy-in-women-with-type-1-and-type-2-diabetes-2002-2003.pdf.

4. Crowther CA, Hiller JE, Moss JR, McPhee AJ, Jeffries WS, Robinson JS, for the Australian Carbohydrate Intolerance Study in Pregnant Women (ACHOIS) Trial Group. Effect of treatment of gestational diabetes mellitus on pregnancy outcomes. N Engl J Med 2005;352:2477-86. http://dx.doi.org/10.1056/NEJMoa042973

5. International Association of Diabetes and Pregnancy Study Groups. Recommendations on the diagnosis and classification of hyperglycemia in pregnancy. Diabetes Care 2010;33:676-82. http://dx.doi.org/10.2337/dc09-1848

6. WHO 2013 Diagnostic Criteria and Classification of Hyperglycaemia First Detected in Pregnancy. Available at http://apps.who.int/iris/bitstream/ 10665/85975/1NHO_NMH_MND_13.2_eng.pdf accessed 1.7.2015

7. Seely EW, Zera C. Gestational Diabetes Mellitus. BMJ Best Practice. http://bestpractice.bmj.com/best-practice/monograph/665/diagnosis/criteria.html as updated Mar 04, 2015

8. American Diabetes Association. Classification and diagnosis of diabetes. Diabetes Care 2015;38(Supplement 1):S8-S16. http://dx.doi.org/10.2337/dc15-S005)

9. Rowan JA, Hague WM, Gao W, Battin MR, Moore MP for the MiG Trial Investigators. Metformin versus insulin for the treatment of gestational diabetes. N Engl J Med 2008;358:2003-15. http://dx.doi.org/10.1056/NEJMoa0707193

10. Murphy J. Unpublished data from internal audit. Birmingham Women's Hospital 2014

11. O'Connor C, O'Shea PM, Owens LA, et al. Trimester-specific reference intervals for haemoglobin A1c (HbA1c) in pregnancy. Clin Chem Lab Med 2011;50:905-09. http://dx.doi.org/10.1515/CCLM.2011.397

12. National Institute for Health and Care Excellence. Preventing type 2 diabetes: risk identification and interventions for individuals at high risk. PH38, July 2012. Available at https://www.nice.org.uk/guidance/ph38. 